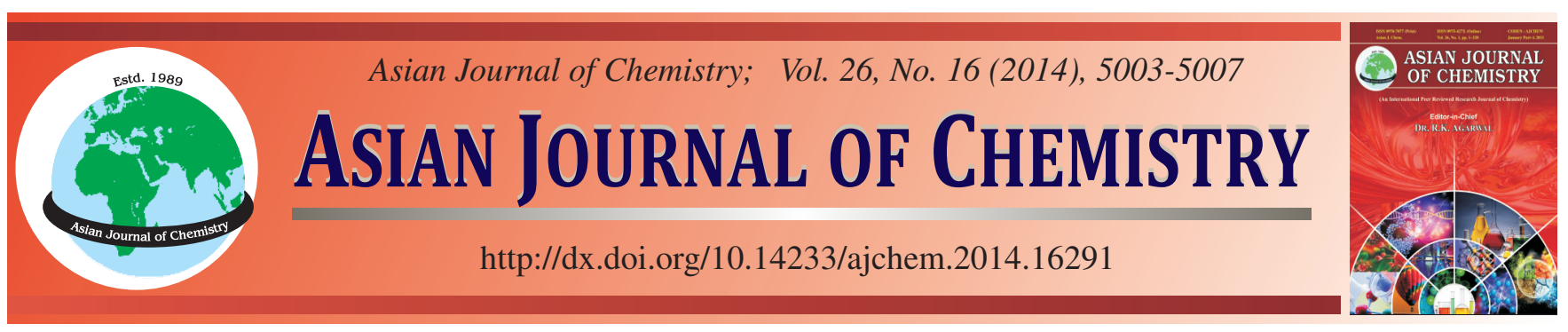

\title{
Synthesis, Growth and Characterization of Nonlinear Optical Ce-Doped L-Prolinium Picrate Single Crystals
}

\author{
T. Gurumurthi ${ }^{1,2}$ and P. Murugakoothan ${ }^{2, *}$
}

${ }^{1}$ Department of Physics, Easwari Engineering College, Chennai-600 089, India

${ }^{2}$ MRDL, PG and Research Department of Physics, Pachaiyappa's College, Chennai-600 030, India

*Corresponding author: E-mail: murugakoothan03@yahoo.co.in

Keywords: Growth from solution, Non-linear optical crystal, X-ray diffraction, Semi organic compound, Micro hardness.

\section{INTRODUCTION}

Non-linear optical (NLO) crystals, due to their non-linear properties, have been found to have enormous applications in frequency conversion, image processing, data storage and fiber optical communication etc. Rare earth ions introduced as dopant during the crystal growth have important consequences on the growth kinetics, morphology and quality of crystals, improving in many cases their physical properties for specific applications. Efforts have been made to improve the NLO properties of the L-prolinium picrate crystal by doping with various rare earth elements. The growth, optical absorption and mechanical studies on pure L-prolinium picrate were studied and reported $^{1,2}$.

The availability of the rare earth elements is of great interest to scientists doing basic research. In the present investigation, attempts were made to study the role of Cerium as dopant on growth, physical, mechanical and optical properties, thermal, dielectric and hardness of pure L-prolinium picrate.

\section{EXPERIMENTAL}

Commercially available L-proline and picric acid were mixed in stoichiometric ratio and dissolved in the mixed solvent of double distilled water and acetone in $1: 1$ ratio to synthesize
L-proline picrate (LPP) source material. The synthesized salt was purified by repeated recrystallisation process and used for growing pure L-prolinium picrate. Cerium doped Lprolinium picrate crystal was grown from mixed solvents of water and acetone in the ratio of 1:1 using the well known solvent evaporation technique with 5 and $10 \mathrm{~mol} \%$ of Cerium nitrate added to the L-prolinium picrate saturated solutions.

Optical quality crystals were collected in a period of 35 days from the supersaturated solution and are shown in Fig. 1. From the physical observations of the grown crystals the 5 mol \% cerium doped L-prolinium picrate (Ce5 : L-prolinium picrate) crystals have good transparency and morphology.

Characterization: The grown single crystals of pure and Ce5 : L-prolinium picrate were subjected to X-ray diffraction studies using a Bruker AXS Kappa APEX II single crystal CCD diffractometer equipped with graphite monochromated $\operatorname{Mo}\left(\mathrm{K}_{\alpha}\right)(\lambda=0.7107 \AA)$. EDAX studies was carried out using EDAX-AMETEK tester for the grown sample to analyze the percentage of dopants in the crystal. HIOKI 3532-50 LCR HITESTER was used for the dielectric study. The sample of size $4 \mathrm{~mm} \times 4 \mathrm{~mm} \times 3 \mathrm{~mm}$ was prepared and mounted between copper electrodes. In order to ensure good electrical contact between the crystal and the electrodes, the crystal faces were coated with silver paint. The dielectric measurements were carried out in a frequency range $100 \mathrm{~Hz}-5 \mathrm{MHz}$ and temperature 


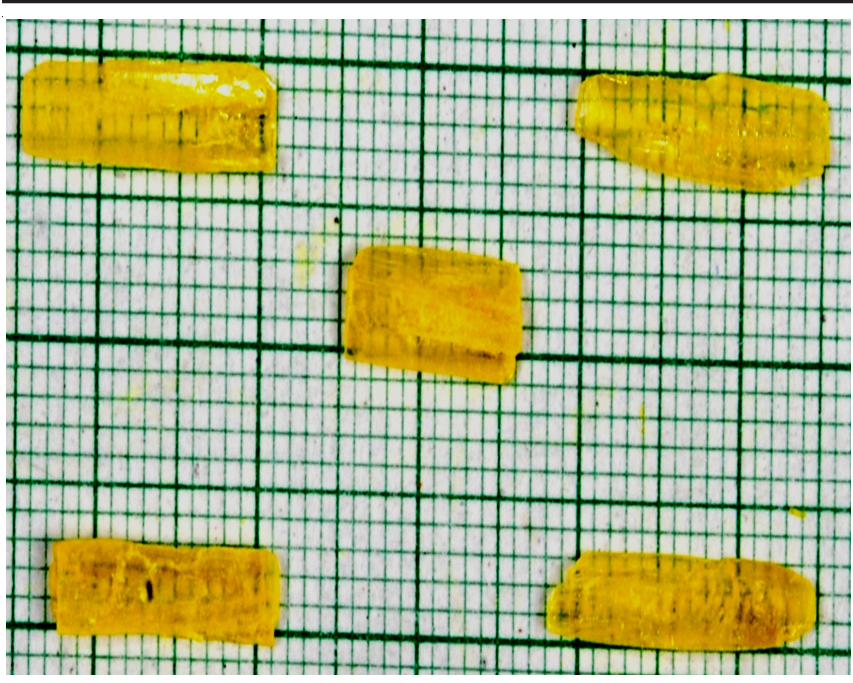

Fig. 1. As grown crystals of $5 \mathrm{~mol} \% \mathrm{Ce}$ doped L-prolinium picrate (Ce5 : L-prolinium picrate)

range $35-95^{\circ} \mathrm{C}$. The Thermogravimetric and differential thermal analyses (TG-DTA) response curves were drawn for pure and Ce5 : L-prolinium picrate powder sample in the temperature range from 20 to $1400{ }^{\circ} \mathrm{C}$ using the instrument NETZSCH STA $409 \mathrm{C}$ at the heating rate of $10 \mathrm{~K} / \mathrm{min}$. in nitrogen atmosphere. The optical transmission spectra were recorded using Shimadzu model-1601 in the wavelength range of 300-1200 $\mathrm{nm}$. The study of NLO conversion efficiency was carried out by the powder technique of Kurtz and Perry. The sample was ground into fine powder and tightly packed in a micro capillary tube. It was mounted in the path of the laser beam. A Q-switched flash lamp pumped $\mathrm{Nd}$ :YAG laser of power $3.2 \mathrm{~mJ}$ with a wavelength of $1064 \mathrm{~nm}$, pulse duration of $8 \mathrm{~ns}$, a repetition rate of $10 \mathrm{~Hz}$ and a spot size of $1 \mathrm{~mm}$ diameter was used for SHG test. Vickers hardness study was made on the as grown crystal using Leica-reichert polyvar2 model hardness tester fitted with a diamond indentor. Etching studies of the Ce5 : Lprolinium picrate single crystal was carried out by REICHERT POLYVAR 2-Metallurgical microscope using water-acetone as an etchant.

\section{RESULTS AND DISCUSSION}

Single crystal X-ray diffraction: Lattice parameter values of pure L-prolinium picrate (LPP) and Ce5 : L-prolinium picrate single crystals are listed in Table-1. The observed values for L-prolinium picrate are in good agreement with the reported values $^{1,2}$.

\begin{tabular}{ccc}
\multicolumn{3}{c}{ TABLE 1 } \\
\multicolumn{3}{c}{ COMPARISON OF SINGLE CRYSTAL X-RAY DATA } \\
OF L-PROLINIUM PICRATE AND Ce5 : L-PROLINIUM \\
PICRATE CRYSTALS \\
\hline \multirow{3}{*}{ Parameters } & L-Prolinium picrate & Ce5 : L-prolinium \\
picrate \\
\hline $\mathrm{a}(\AA)$ & 10.902 & 10.825 \\
$\mathrm{~b}(\AA)$ & 5.352 & 5.432 \\
$\mathrm{c}(\AA)$ & 12.472 & 12.224 \\
$\mathrm{~V}\left(\AA^{3}\right)$ & 687.60 & 682.32 \\
System & Monoclinic & Monoclinic \\
$\beta\left({ }^{\circ}\right)$ & 109.11 & 109.14 \\
Space group & $\mathrm{P}_{21}$ & $\mathrm{P}_{21}$ \\
\hline
\end{tabular}

EDAX analysis: In order to analyze quantitatively the presence of cerium in the crystal, EDAX study was carried out for the grown sample and the percentage of dopant present in the L-prolinium picrate was confirmed and tabulated in Table-2. From EDAX analysis, it is observed that the amount of cerium atoms present inside the L-prolinium picrate lattice is very less.

TABLE 2

EDAX ANALYSIS OF CE5 : L-PROLINIUM PICRATE CRYSTAL

\begin{tabular}{ccc}
\hline Element & Wt. \% & At. \% \\
\hline $\mathrm{Ce}$ & 3.42 & 0.47 \\
\hline
\end{tabular}

Dielectric studies: The dielectric constant and dielectric loss were calculated for pure and doped crystals. Fig. 2a and $2 \mathrm{~b}$ show the variation of dielectric constant and dielectric loss as a function of frequency. It is found that the dielectric constants of L-prolinium picrate and doped L-prolinium picrate crystals are high at lower frequencies and they decrease with increase in frequency. The trend of the dielectric constants of L-prolinium picrate and $5 \mathrm{~mol} \% \mathrm{Ce}$ doped L-prolinium picrate crystals are almost the same. But at fixed frequency, the
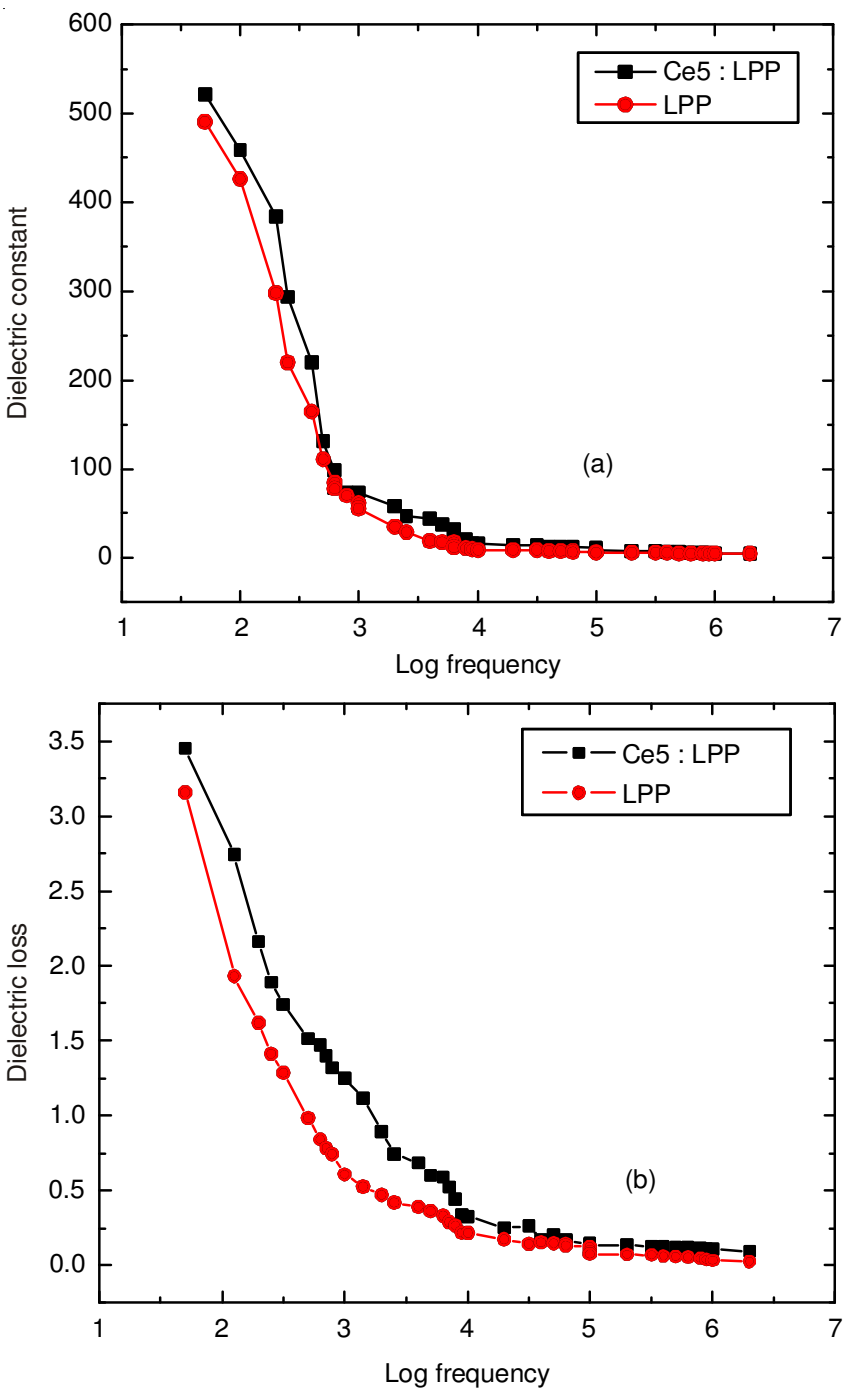

Fig. 2. (a) Dielectric constant vs. log frequency for pure and doped Lprolinium picrate crystals; (b) Dielectric loss vs. log frequency for pure and doped L-prolinium picrate crystals 
dielectric constants of doped L-prolinium picrate crystals are less than that of pure one. In accordance with Miller rule, the lower value of dielectric constant is a suitable parameter for the enhancement of second harmonic generation (SHG) coefficient $^{3}$. Since the dielectric constant of doped crystals is lower than that of the pure crystals they are less polarized in comparison with pure crystal. The lower polarization may be due to ineffective transportation of polarization from one molecule to its neighbor in the presence of dopants ${ }^{4}$.

The characteristic of low dielectric loss at high frequencies for these samples suggest that the pure and doped crystals possess enhanced quality with lesser defects ${ }^{5}$. For a particular frequency, the dielectric loss of doped L-prolinium picrate is slightly lesser than that of pure, which indicates that the dopant enhances the optical quality of L-prolinium picrate and reduces the defects.

Thermal analyses: The TG - DTA curves of L-prolinium picrate and Ce5 : L-prolinium picrate samples exhibits nearly similar stage of decomposition between 100 and $1200{ }^{\circ} \mathrm{C}$ as shown in Fig. 3(a) and 3(b). In order to study the influence of the dopant on the thermal stability of L-prolinium picrate, the temperature corresponding to a peak maximum of first stage of phase transition in DTA trace is taken into account for comparison. The temperature on the first state of transition for the L-prolinium picrate is found at $158^{\circ} \mathrm{C}$ and for $\mathrm{Ce} 5$ : Lprolinium picrate it is found at $162{ }^{\circ} \mathrm{C}$. This shows that the doped crystal possess better thermal stability compared to pure crystal.
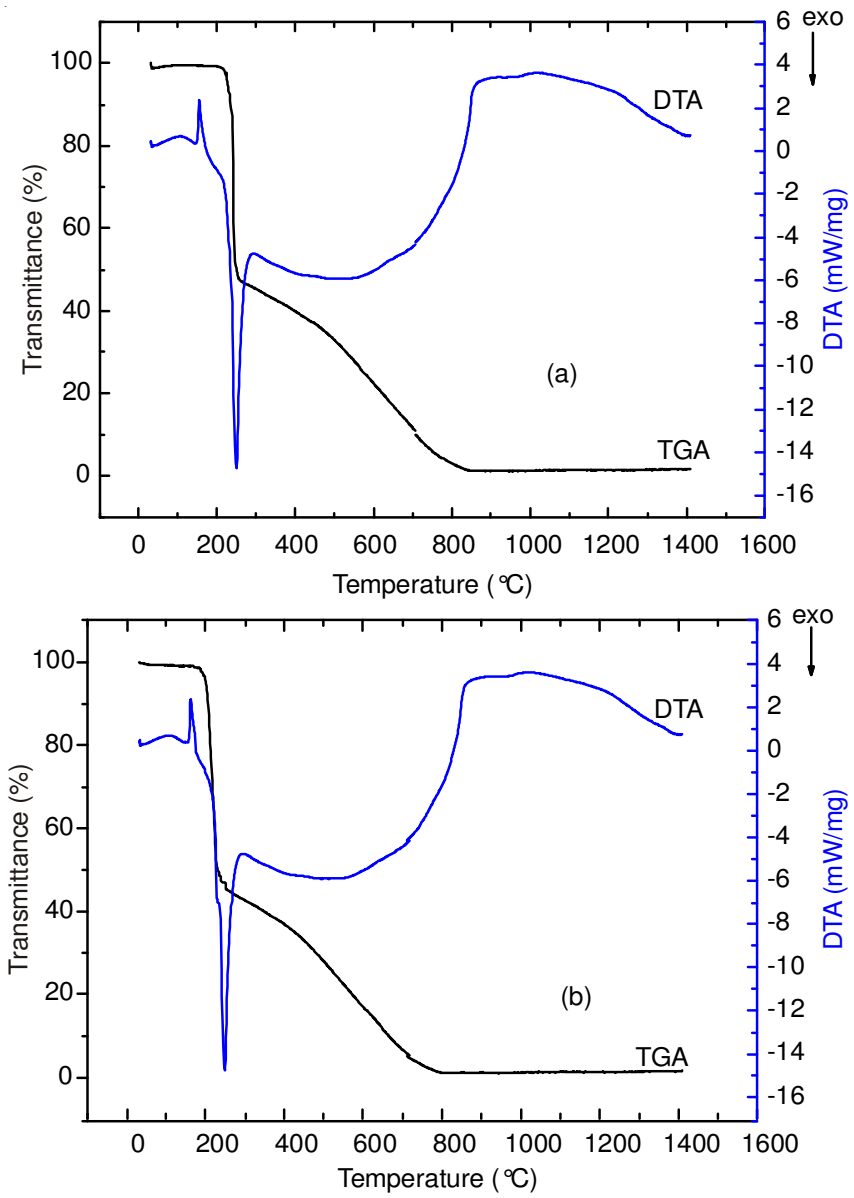

Fig. 3. (a) TG-DTA graph for L-prolinium picrate sample; (b) TG-DTA graph for Ce5 : L-prolinium picrate sample
Optical transmission study: Fig. 4 shows the optical transmission spectra of L-prolinium picrate and Ce5 : L-prolinium picrate crystals. The thickness of the sample used for this study was $1.5 \mathrm{~mm}$. It is found that L-prolinium picrate crystal has transmittance of $66 \%$ and Ce5 : L-prolinium picrate crystal has the transmittance of $63 \%$. The lower cut off for Lprolinium picrate and $\mathrm{Ce} 5$ : L-prolinium picrate crystals are $470 \mathrm{~nm}$. This shows that doping the crystal with cerium did not shift the lower cut-off value, but, the percentage of transmission is decreased due to cerium addition.

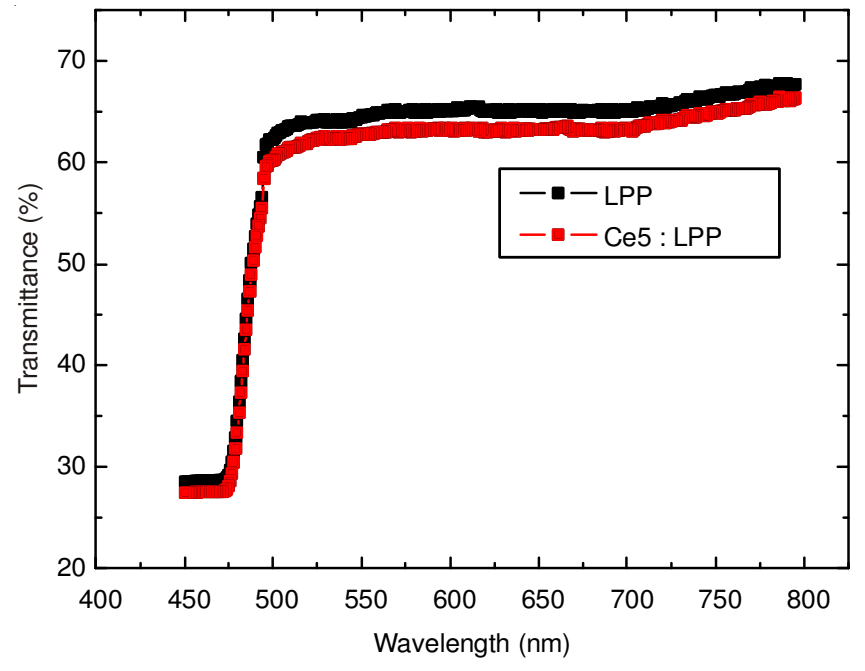

Fig. 4. Optical transmission for L-prolinium picrate and Ce5 : L-prolinium picrate crystals

Powder second harmonic generation measurement: The study of NLO conversion efficiency was carried out by the powder technique of Kurtz and Perry ${ }^{6}$. Second harmonic signal of $474 \mathrm{mV}$ was obtained for an input energy of $3.2 \mathrm{~mJ} /$ pulse for Ce5 : L-prolinium picrate. But the standard KDP sample gave a second harmonic generation signal of $15 \mathrm{mV} /$ pulse for the same input energy. The results obtained by this method shows that second harmonic generation efficiency of Ce5 : L-prolinium picrate is nearly 31.6 times higher than that of KDP but pure L-prolinium picrate shows the second harmonic generation efficiency of 48 times higher than that of KDP and this is due to ineffective transportation of polarization from one molecule to its neighbor in the presence of dopants for Ce5 : L-prolinium picrate. Since the second order non linear efficiency will vary with the particle size of powder sample ${ }^{7}$, the care has been taken to maintain uniform particle size of source and the reference material.

Vicker's microhardness study: The good quality crystals are needed not only with good optical performance but also with good mechanical behaviour ${ }^{8}$ for applications. In order to study the mechanical behaviour of the grown L-prolinium picrate crystal, indentations were made on the cleaved (100) plane of pure L-prolinium picrate and Ce 5 : L-prolinium picrate crystals with the applied load ranging from 5 to $100 \mathrm{~g}$. The time of indentation was kept constant as $5 \mathrm{~s}$ for all indentations. The Vicker's hardness number was calculated using the relation ${ }^{9}$.

$$
\mathrm{H}_{\mathrm{V}}=\frac{1.854 \mathrm{P}}{\mathrm{d}^{2}} \mathrm{~kg} / \mathrm{mm}^{2}
$$


where $\mathrm{P}$ is the applied load and $\mathrm{d}$ is the diagonal length. The Vicker's hardness for L-prolinium picrate and Ce5:L-prolinium picrate crystals as a function of load are shown in Fig. 5. The hardness values of L-prolinium picrate have been found to be lower than that of Ce5:L-prolinium picrate crystal. Vicker's hardness increases with increase of load till $60 \mathrm{~g}$ for Ce $5: \mathrm{L}-$ prolinium picrate crystal but upto $50 \mathrm{~g}$ for L-prolinium picrate crystal. The Loads above $60 \mathrm{~g}$ for Ce5: L-prolinium picrate and $50 \mathrm{~g}$ for L-prolinium picrate developed multiple cracks around the indentation mark and hardness decreases with the further increase of load. By plotting $\log \mathrm{P}$ versus $\log \mathrm{d}$, the value of the work hardening coefficient (n) was found to be 3.73 for L-prolinium picrate and 3.01 for Ce5 : L-prolinium picrate. According to onitsch, $1 \leq \mathrm{n} \leq 1.6$ for hard materials and $n>1.6$ for soft materials ${ }^{10}$. Hence it is concluded that Lprolinium picrate and Ce5:L-prolinium picrate are soft materials. In order to find the increase in strength that accompanies plastic deformation of the grown crystal, yield strength $\left(\sigma_{y}\right)$ of the crystals was also calculated using the relation ${ }^{11}$

$$
\sigma_{\mathrm{y}}=\frac{\left(\mathrm{H}_{\mathrm{V}}\right)}{3} 0.1^{\mathrm{n}-2} \mathrm{MPa}
$$

where ' $\mathrm{H}_{\mathrm{v}}$ ' is the maximum hardness and ' $\mathrm{n}$ ' is the work hardening coefficient. Yield strength for L-prolinium picrate and Ce5 : L-prolinium picrate crystals are found to be $0.07 \mathrm{MPa}$ and $0.08 \mathrm{MPa}$, respectively.

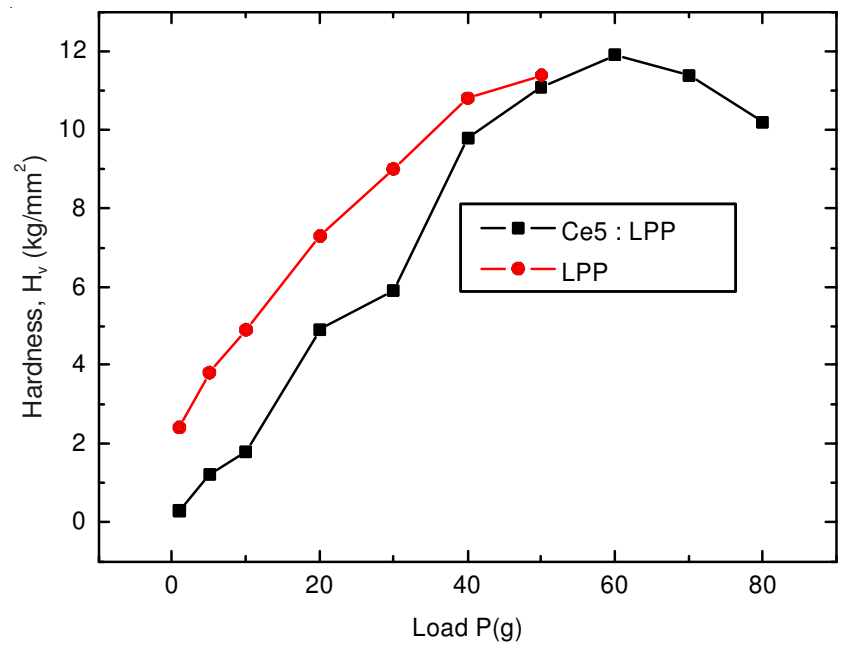

Fig. 5. Micro hardness value of L-prolinium picrate and Ce5 : L-prolinium picrate crystals
Moreover while analyzing the optical image of the indentation in the crystal for L-prolinium picrate and Ce5 : Lprolinium picrate, it is observed that mild crack started at $10 \mathrm{~g}$ load for both L-prolinium picrate and Ce5 : L-prolinium picrate and multicracks developed at $70 \mathrm{~g}$ load for Ce5 : L-prolinium picrate and $50 \mathrm{~g}$ load for L-prolinium picrate. Crack length was measured for various forces applied on the crystal. It was about $35 \mu \mathrm{m}$ length from the indentation center for the load between 10 and $50 \mathrm{~g}$. There were no remarkable changes found in the indentation length and crack length in this range of load. But when the applied load reached $80 \mathrm{~g}$, the crystal was found to be deformed more, which can be seen in the optical image shown in the Fig. 6. Elastic stiffness constant was calculated ${ }^{4,12}$ from the microhardness by Wooster's empirical relation $\mathrm{C}_{11}=$ $\mathrm{H}_{\mathrm{v}}{ }^{7 / 4}$. The maximum stiffness constants for the L-prolinium picrate and Ce5:L-prolinium picrate crystals are $67.36 \times 10^{12}$ and $76.24 \times 10^{12}$ Pascals, respectively. Fracture toughness $\left(\mathrm{K}_{\mathrm{c}}\right)$ is the resistance of a material to failure from fracture starting from a preexisting crack. It was calculated using the formula $\mathrm{K}_{\mathrm{c}}=\mathrm{P} / \beta \mathrm{C}^{3 / 2}$, where $\mathrm{C}$ is the crack length from the center of the indentation, $\mathrm{P}$ is the applied load and $\beta(=7)$ is the geometrical constant for Vicker's indenter ${ }^{13}$. The crack length developed with maximum hardness at $60 \mathrm{~g}$ applied load for Ce:Lprolinium picrate crystal was $43 \mu \mathrm{m}$. Hence the fracture toughness was calculated as $30,398 \mathrm{~kg} \mathrm{~m}^{-3 / 2}$. Brittleness is an important property of the crystal which determines its fracture without any appreciable deformation. It is expressed in terms of brittleness index ${ }^{14}$. Brittleness index was calculated ${ }^{2,15}$ using the formula $\mathrm{B}_{\mathrm{i}}=\mathrm{H}_{\mathrm{v}} / \mathrm{K}_{\mathrm{c}}$ as $3.552 \times 10^{-3} \mathrm{~m}^{-1 / 2}$.

Fig. 7a shows the as grown crystal surface and Fig. $7 \mathrm{~b}$ shows the etch patterns produced for $5 \mathrm{~s}$. Etching produces triangular shaped etch pits. It is observed that etch pit is symmetrical in nature. The possible reason for the formations of these etch pits is due to the impurity in the crystal during growth, which introduces strain in the crystal lattice and serves as sites of dissolution ${ }^{16}$.

\section{Conclusion}

L-Prolinium picrate and cerium doped L-prolinium picrate crystals were grown from mixed solvent of water and acetone in the ratio of $1: 1$ by the solvent evaporation method. From the XRD analysis, it is observed that the L-prolinium picrate and Ce doped L-prolinium picrate crystals retain the monoclinic structure and the calculated lattice parameter values

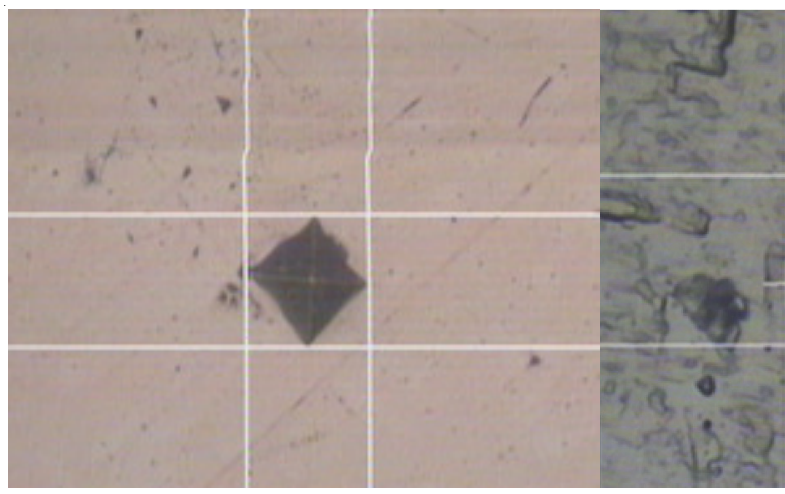

(a)

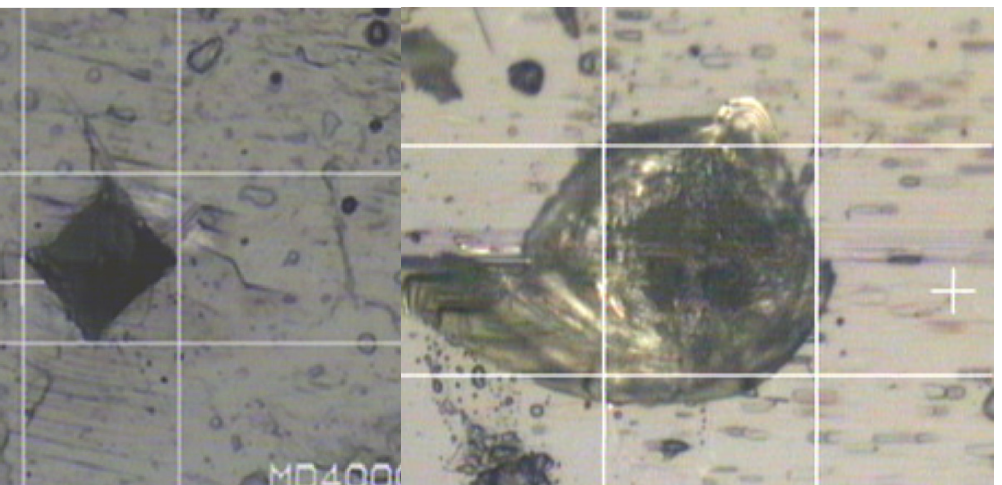

(b) (c)

Fig. 6. Indentation image of Ce5 : L-prolinium picrate crystal for; (a) $5 \mathrm{~g}$ (b) $50 \mathrm{~g}$ and (c) $80 \mathrm{~g}$ 

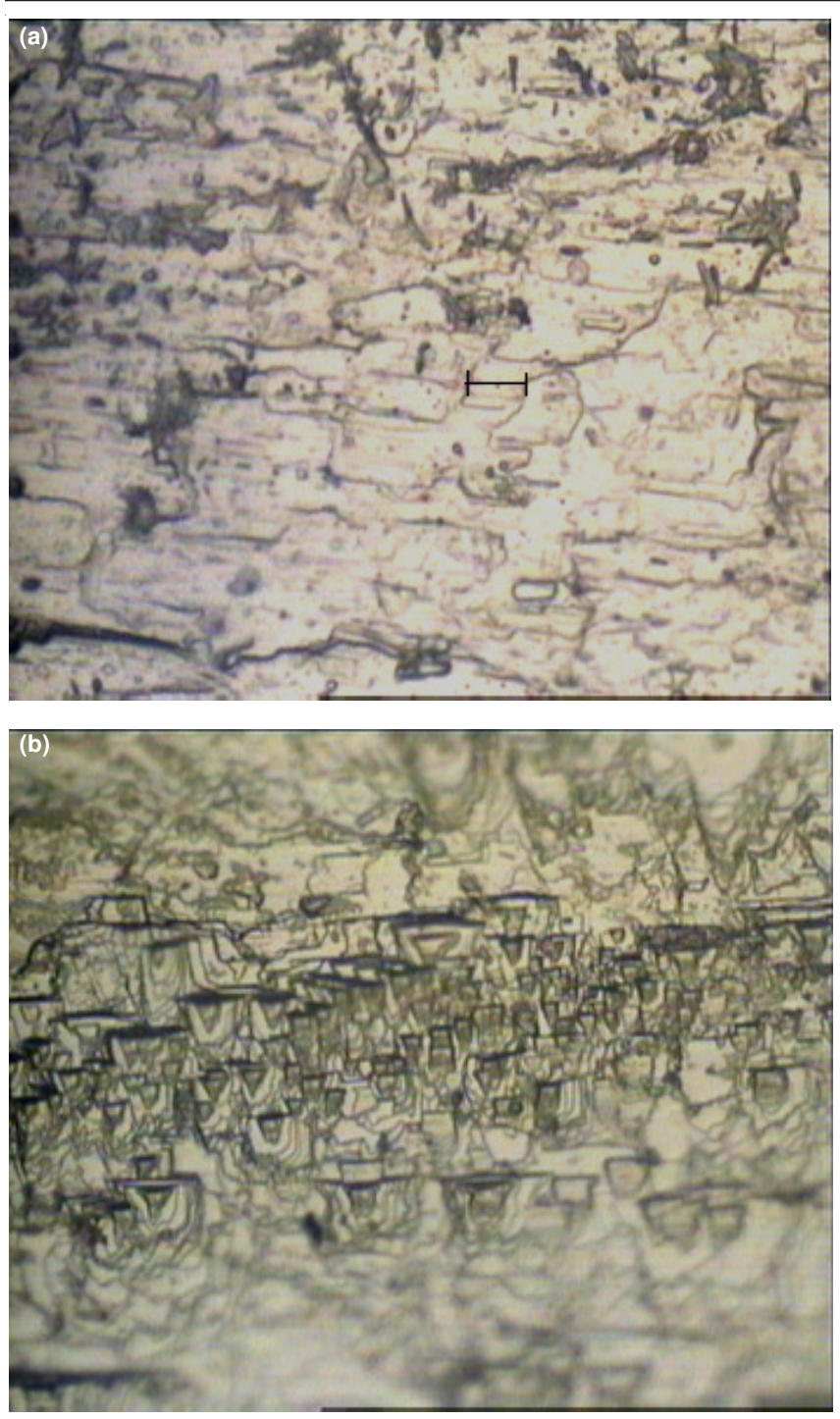

Fig. 7. (a) Surface of the as grown Ce : L-prolinium picrate (b) Etch patterns produced for $5 \mathrm{sec}$

are comparable with the reported values of L-prolinium picrate. The presence of cerium in L-prolinium picrate crystal was confirmed by EDAX analysis. Optical transmission study shows that the grown cerium doped L-prolinium picrate crystal has high transparency in the wavelength range from 470 to $800 \mathrm{~nm}$. The dielectric constant and dielectric loss of Ce doped
L-prolinium picrate is found to be lesser than that of L-prolinium picrate. This shows that the doped crystal possess better optical quality with lesser defects compared to pure crystals. The thermal studies of the samples suggest that the thermal stability is better for doped crystals. Hardness study reveals that the L-prolinium picrate and Ce $5:$ L-prolinium picrate crystals are soft materials. Higher hardness is obtained for Ce5 : Lprolinium picrate than that of the L-prolinium picrate crystals. Yield strength, elastic stiffness, fracture toughness and brittleness index of the Ce5 : L-prolinium picrate crystals were also reported. The NLO efficiency for Ce5 : L-prolinium picrate crystal is 31.6 times higher than that of KDP crystals.

ACKNOWLEDGEMENTS

The authors acknowledged Prof. P.K. Das, Department of IPC, IISc, Bangalore, Prof. V. Ravichandran, Head of the Department, Nuclear Physics, University of Madras and IITSAIF, Chennai for carrying out the characterization of the sample.

\section{REFERENCES}

1. S.A. Martin Britto Dhas, G. Bhagavannarayana and S. Natarajan, $J$. Cryst. Growth, 310, 3535 (2008).

2. T.U. Devi, N. Lawrence, R.R. Babu and K. Ramamurthi, J. Cryst. Growth, 310, 116 (2008).

3. U. Von Hundelshausen, Phys. Lett. A, 34, 405 (1971).

4. T.K. Kumar, S. Janarthanan, M.V.A. Raj, S. Pandi, P. Sagayaraj and D.P. Anand, J. Phys. Chem. Solids, 69, 2634 (2008).

5. C. Balarew and R. Duhlev, J. Solid State Chem., 55, 1 (1984).

6. S.K. Kurtz and T.T. Perry, J. Appl. Phys., 39, 3798 (1968).

7. Y. Porter, K.M. Ok, N.S.P. Bhuvanesh and P.S. Halasyamani, Chem. Mater., 13, 1910 (2001).

8. M.K. Marchewka, S. Debrus and H. Ratajczak, Cryst. Growth Des., 3, 587 (2003).

9. A. Rahman and J. Podder, Indian J. Phys., 86, 15 (2012).

10. J. Madhavan, S. Aruna, K. Prabha, J.P. Julius, G.P. Joseph, S. Selvakumar and P. Sagayaraj, J. Cryst. Growth, 293, 409 (2006).

11. M.E. Peter and P. Ramasamy, J. Cryst. Growth, 312, 1952 (2010).

12. S. Suresh, A. Ramanand, P. Mani and K. Murthy, J. Optoelectron. Biomed. Mater., 1, 129 (2010).

13. S. Krishnan, C. Justin Raj, R. Robert, A. Ramanand and S. Jerome Das, Solid-State Electron., 52, 1157 (2008).

14. M. Bhat, B. Kaur, K.K. Bamzai, P.N. Kotru and B.M. Wanklyn, J. Phys. Chem. Solids, 65, 1359 (2004).

15. G. Anandha Babu, G. Bhagavannarayana and P. Ramasamy, J. Cryst. Growth, 310, 2820 (2008).

16. S. Mukerji and T. Kar, J. Cryst. Growth, 204, 341 (1999). 